\title{
Silicon nanobridge as a high quality mechanical resonator.
}

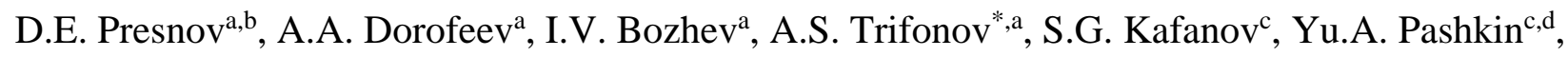 \\ V.A. Krupenin ${ }^{\mathrm{a}}$ \\ ${ }^{a}$ Quantum Technology Centre, Faculty of Physics, M.V. Lomonosov Moscow State University, \\ Moscow, 119991, Russia.; ${ }^{b}$ D.V. Skobeltsyn Institute of Nuclear Physics, M.V. Lomonosov \\ Moscow State University, Moscow, 119991, Russia.; 'Department of Physics, Lancaster University, \\ Lancaster, LA1 4YB, United Kingdom; 'Lebedev Physical Institute, Moscow 119991, Russia.
}

\begin{abstract}
The paper presents details of the fabricating technology of nanoscale mechanical resonators based on suspended silicon nanowires. The structures were made from silicon on insulator material, the thickness of the upper layer of silicon is 110 $\mathrm{nm}$, the thickness of silicon oxide is $200 \mathrm{~nm}$. Fabrication process contains standard CMOS compatible technologies only: electron lithography with positive resist, reactive ion and liquid etching, electron beam deposition of thin films. The presented structures can be used as sensors of mass, displacement, acceleration, pressure with extremely high sensitivity.
\end{abstract}

Keywords: Nanoelectromechanical systems (NEMS), nanowire, nanoresonator, solicon-on-insulator (SOI). 


\section{INTRODUCTION}

Nanoelectromechanical systems (NEMS) have been actively developed and investigated in the last decade. These systems are of great interest from both fundamental and applied points of view [1-4]. NEMS structures are extremely promising for creating ultrasensitive detectors of mass [5, 6], force [7], pressure [8], and displacement [9, 10]. Combination of nanomechanical resonators with optical and electronic converters make it possible to study different quantum effects [11-13]. Also, small-size mechanical resonators are a very effective tool in understanding the properties of superfluids [14].

In recent years, suspended silicon nanostructures have attracted the attention of scientists and technologists as a structure for fabrication various types of switches [15]. Using such structure as a switch it is possible to decrease the response time significantly and to increase the speed and efficiency of the device, comparing with existing analogues. Also, these switches can be used as functional elements in quantum computational networks. In [16], it was demonstrated suspended silicon structure, which was used as generator of a physical unclonable function. This function is an alternative method of coding information. The small size of such generators makes it extremely attractive for data encryption in personal devices.

Silicon on insulator (SOI) is a very promising material for the fabrication of nanoelectromechanical systems [17], solidstate single atom structures [18] and sensors based on field-effect transistors with nanowire channel [19, 20]. The SOI is a three-layer material, in which the upper thin layer of monocrystalline silicon is separated from the handle substrate by a thin layer of silicon oxide. In [21], silicon nanomechanical resonators with a record quality factor and operating in both linear and nonlinear modes were presented. Here, the fabrication technology of such nanomechanical resonators is described in detail.

\section{STRUCTURES FABRICATION DETAILS}

SOI wafers with $110 \mathrm{~nm}$ thick upper layer of silicon, separated from the base silicon substrate by a silicon dioxide of 200 $\mathrm{nm}$ thick were used for experimental samples fabrication. The technological process is similar to that used to fabrication of sensors based on field-effect transistors with a nanowire channel [19, 20, 22]. The idea of the method is as follows: a nanoscale wire is cut out in the upper layer of SOI, then it is suspended by removing of silicon oxide separating the upper and lower layers of silicon.

Before the fabrication process, the initial SOI wafer (Fig. 1(a)) was washed for 10 seconds in 6\% buffered solution of hydrofluoric acid $\left(\mathrm{NH}_{4} \mathrm{~F} \cdot \mathrm{HF}\right)$ to remove the native oxide from the silicon surface and clean whole wafer before covering it by resists. 
(a)

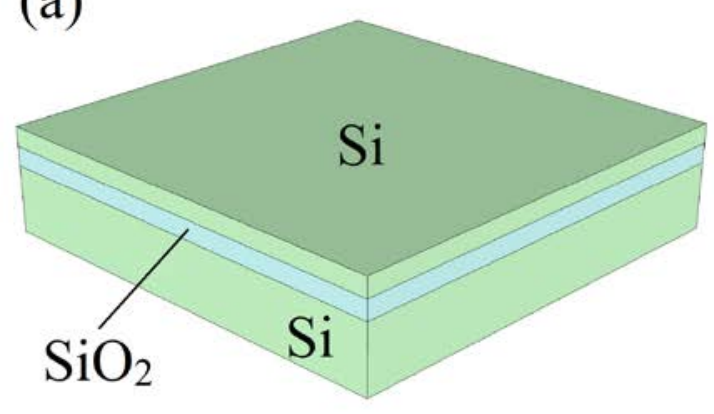

(c)

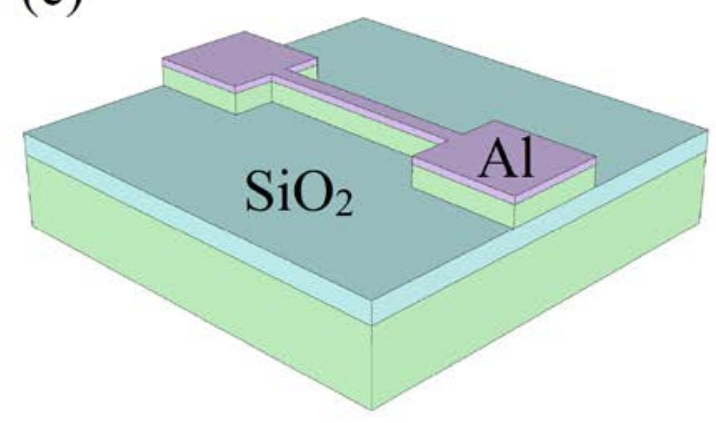

(e)

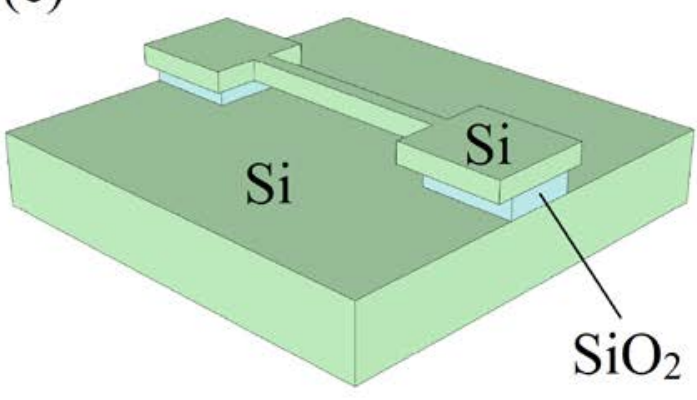

(b)

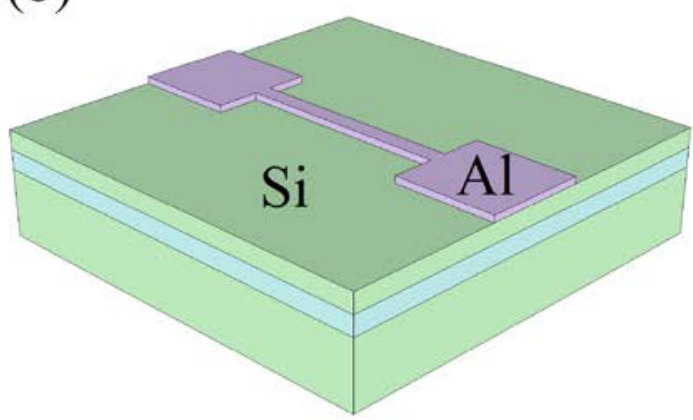

(d)

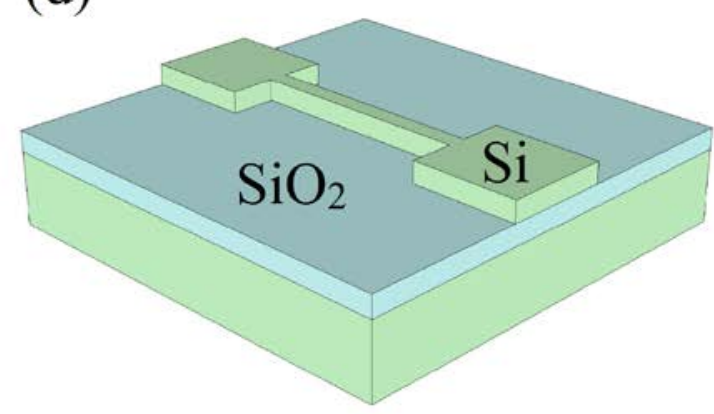

(f)

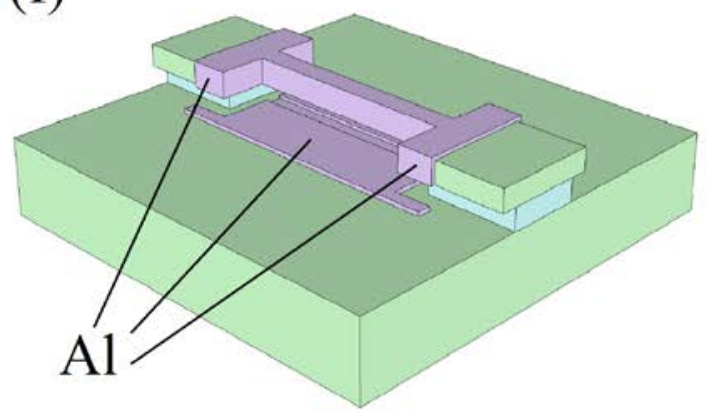

Figure 1. A sequential diagram of the main fabricating stages of suspended silicon nanowires. (a) - initial wafer of silicon on insulator. (b) - deposition of an aluminum mask on the SOI surface. (c) - reactive ion etching of the upper SOI layer through the mask. (d) - structure after removing the aluminum mask in a weak alkaline solution. (e) - liquid etching of a layer of silicon oxide. (f) the final structure.

At the first step, macro-dimensional contacts were fabricated. The lead electrodes structure was exposed using deep UV optical lithography in a double-layer PMMA/MAA positive resist. The resist was spinned at 4000 rpm for 90 seconds for the MAA copolymer (EL11) and 3500 rpm, 30 seconds for the upper layer of PMAA-950K (A4). The first MMA layer was baked at $180{ }^{\circ} \mathrm{C}$ for 20 minutes. The final baking was done at a $160{ }^{\circ} \mathrm{C}, 10$ minutes. As a result, the total mask thickness was $\sim 500 \mathrm{~nm}$. Next, the pattern was exposed through a quartz photo mask using a wavelength of $\lambda=210 \mathrm{~nm}$. After development of the exposed areas of the resist in a solution of toluene with isopropanol (1:3), a metal films were deposited on the substrate: layer of titanium (Ti), $20 \mathrm{~nm}$ thick and gold (Au), $50 \mathrm{~nm}$ thick. The deposition method was magnetron sputtering in an argon atmosphere (Ar) at a pressure of $1.2 \times 10^{-2}$ mbar. The formation of the lead contacts 
structure was completed by removing the $\mathrm{Au} / \mathrm{Ti}$ film with not exposed resist layer areas (lift-off). Lift-off process was carried out in acetone at a temperature of $20^{\circ} \mathrm{C}, 10$ minutes.

Further, nanoscale channels were added to the structure by electron-beam lithography technique. The alignment of lead contacts and nanoscale structures in the lithographer was done using pre-formed markers in the contact pads layer. At this stage only a single-layer of PMMA e-beam resist ( $200 \mathrm{~nm}$ thick) was used. The pattern was exposed with a $\sim 300 \mu \mathrm{C} / \mathrm{cm}^{2}$ dose at $20 \mathrm{kV}$ accelerating voltage and $22 \mathrm{pA}$ probe current. After development (1:10 solution of toluene/IPA) a thin layer of aluminum (Al) $\sim 15 \mathrm{~nm}$ thick was deposited on the sample using electron beam deposition technique. This layer forms a mask on the silicon surface to etch the fine silicon structure (Fig. 1(b)). The deposition rate of Al was approximately $3 \AA$ /sec at base pressure $\sim 10^{-7}$ mbar. After forming the mask, the sample surface was cleaned in the lift-off process, removing the rest of resist in acetone. Then, using reactive ion etching in a fluorine-containing ( $\left.\mathrm{SF}_{6}\right)$ plasma at a pressure of $\sim 1 \mathrm{~Pa}$ and power of $50 \mathrm{~W}$, the nanoscale pattern was transferred to the upper silicon layer (Fig. 1 (c)). After this process the $\mathrm{Al}$ mask was removed in a weak alkaline (KOH) solution (Fig. 1 (d)).

The suspension of the formed nanostructure was carried out by removing dioxide layer from under the nanowire. For this, the sample was one more time covered by PMMA/MAA double-layer mask, in the same way as described above. A region in the vicinity of the nanowire was exposed by electron-beam lithography. After the development of the exposed areas of the resist, a silicon oxide was removed under the nanostructure and in its vicinity through the formed window (Fig. 1(e)). Etching was carried out in a liquid of $6 \%$ buffered solution of hydrofluoric acid $\left(\mathrm{NH}_{4} \mathrm{~F} \cdot \mathrm{HF}\right)$. The rest of resist was washed away in acetone.

To provide the electrical conductivity of the silicon structure at low temperatures, a layer of Al $\sim 20 \mathrm{~nm}$ thick was deposited on the surface of the structure. A positive PMMA/MAA double-layer of e-beam resist was spinned on the sample again. The pattern, overlapping the central part of the lead electrodes and a small area in the vicinity of each nanowire were re-exposed. After development, the electron beam evaporation method was used to deposit Al (base pressure $\sim 10^{-7} \mathrm{mbar}$ and a deposition rate $\sim 2 \AA / \mathrm{sec}$ ). The deposition was done at three different angles for more uniformly covering of the upper and side nanowire faces (Fig. 1 (f)). The deposition angles were $30^{\circ}, 0^{\circ}$ and $-30^{\circ}$ relative to surface of the sample. The rest of resist was lifted off in acetone.

Four resonance structures connected in parallel were formed on the chip (Fig. 2(b), an enlarged image of one of the resonators is shown in Fig. 2(a)). Such design allows us to measure all resonators in one experiment, since the resonators do not influence each other due to the significant difference in their resonance frequencies. The disadvantage of design is the fact that the incoming RF current is distributed between all resonators and it does not allow to estimate the magnitude of external driving force quantitatively. To match the wave impedances of electrical cables and connectors with structure on the chip, a coplanar waveguide was formed on the chip. It represents a central signal conductor (20 nm Ti+50 nm Au) $150 \mu \mathrm{m}$ wide, surrounded on both sides by grounded strips (Fig. 2c). 


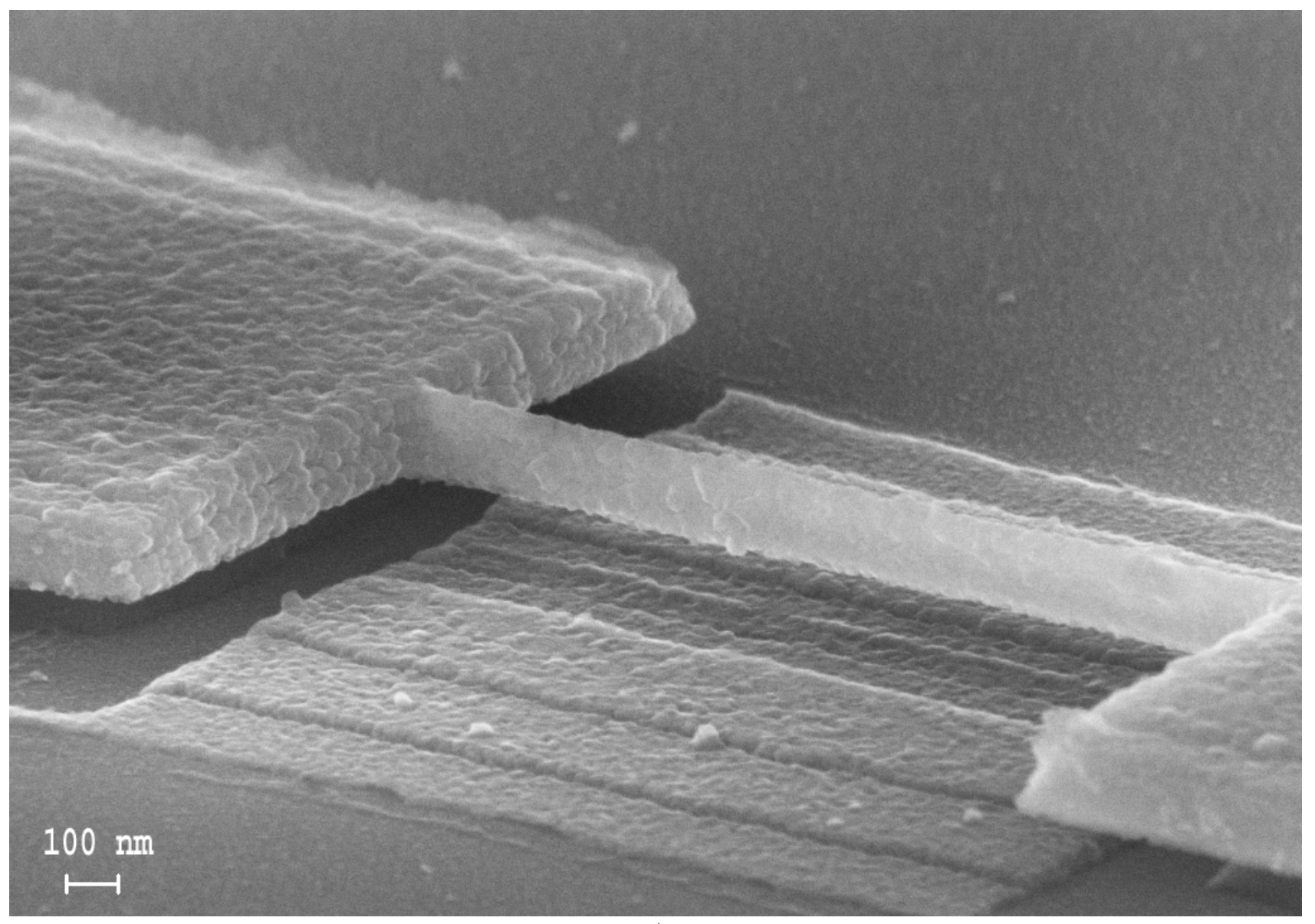

a) 


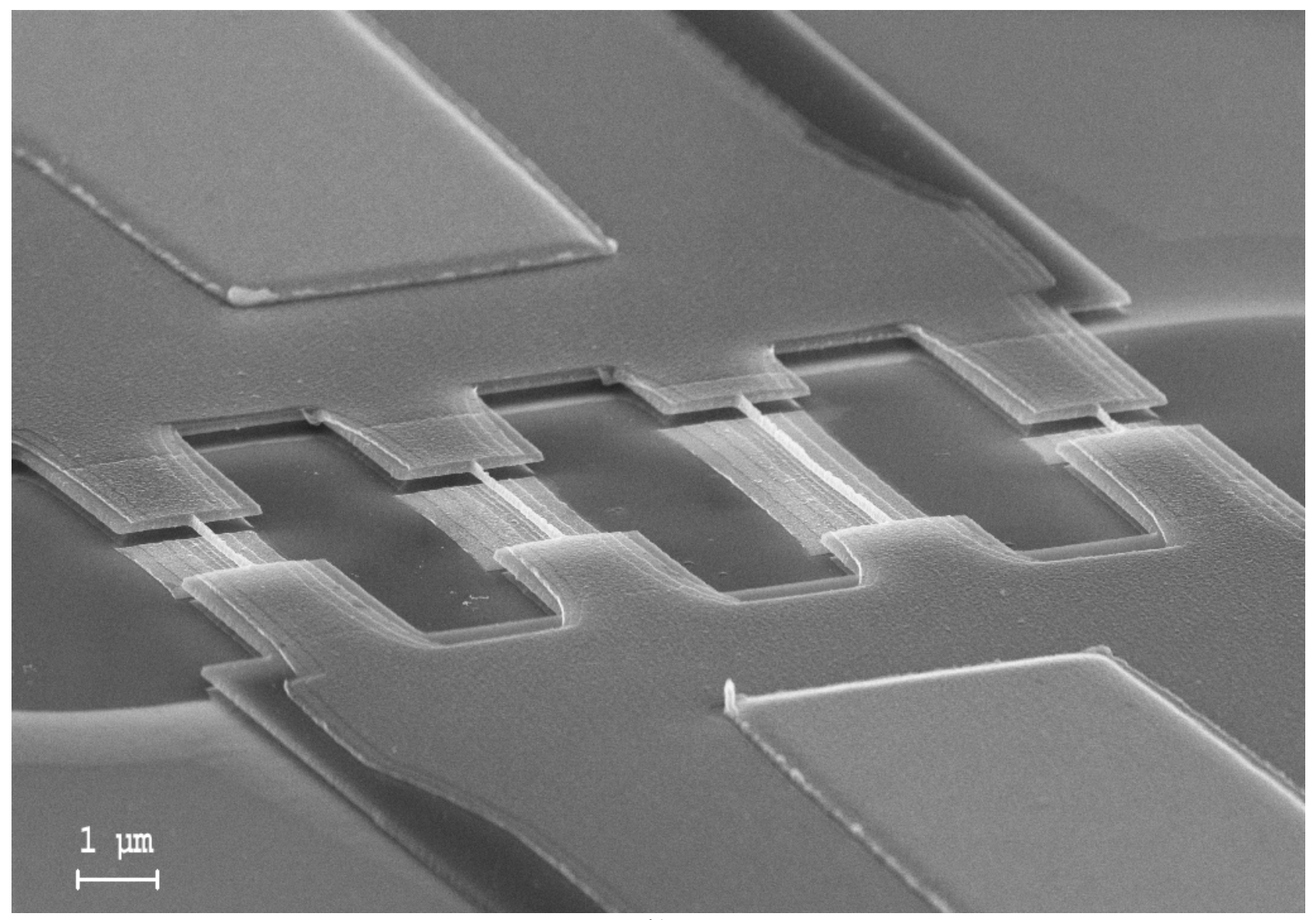

b)

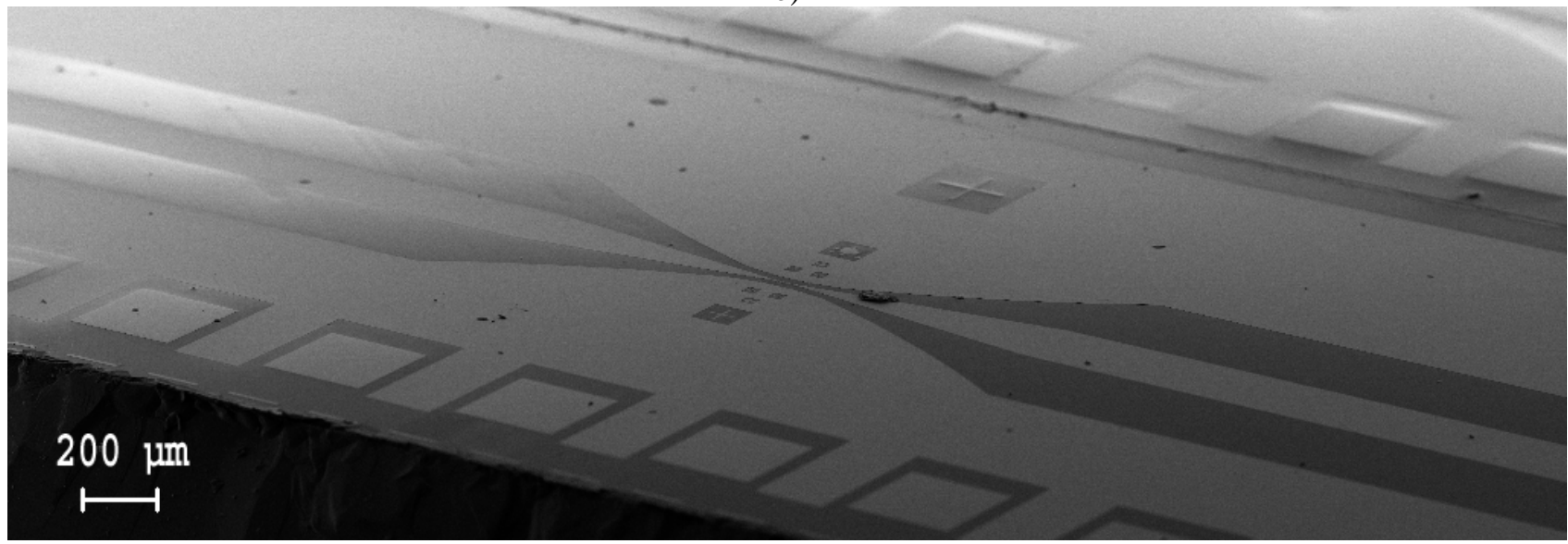

C)

Figure 2. Scanning electron microscope images: a) $2 \mu \mathrm{m}$ length nanoresonator coated with Al film, b) a system of 4 suspended silicon nanowires, c) a coplanar waveguide for connecting microwave signal to the system of suspended silicon nanowires.

The mechanical behavior of the resonators excited by a magnetomotive method [23] are described in detail elsewhere [21]. Fig. 3 shows the dependence of the signal transmission coefficient on the frequency and input power of the external signal fot nanoresonator with a $2 \mu \mathrm{m}$ length, a $85 \mathrm{~nm}$ width and a $110 \mathrm{~nm}$ height. There is a clear region near $32.46 \mathrm{MHz}$ where resonance frequency of the nanowire is visible. The resonance frequency increases at high input power values, indicating nonlinear mode of the resonator operation and increasing of resonator hardness. 


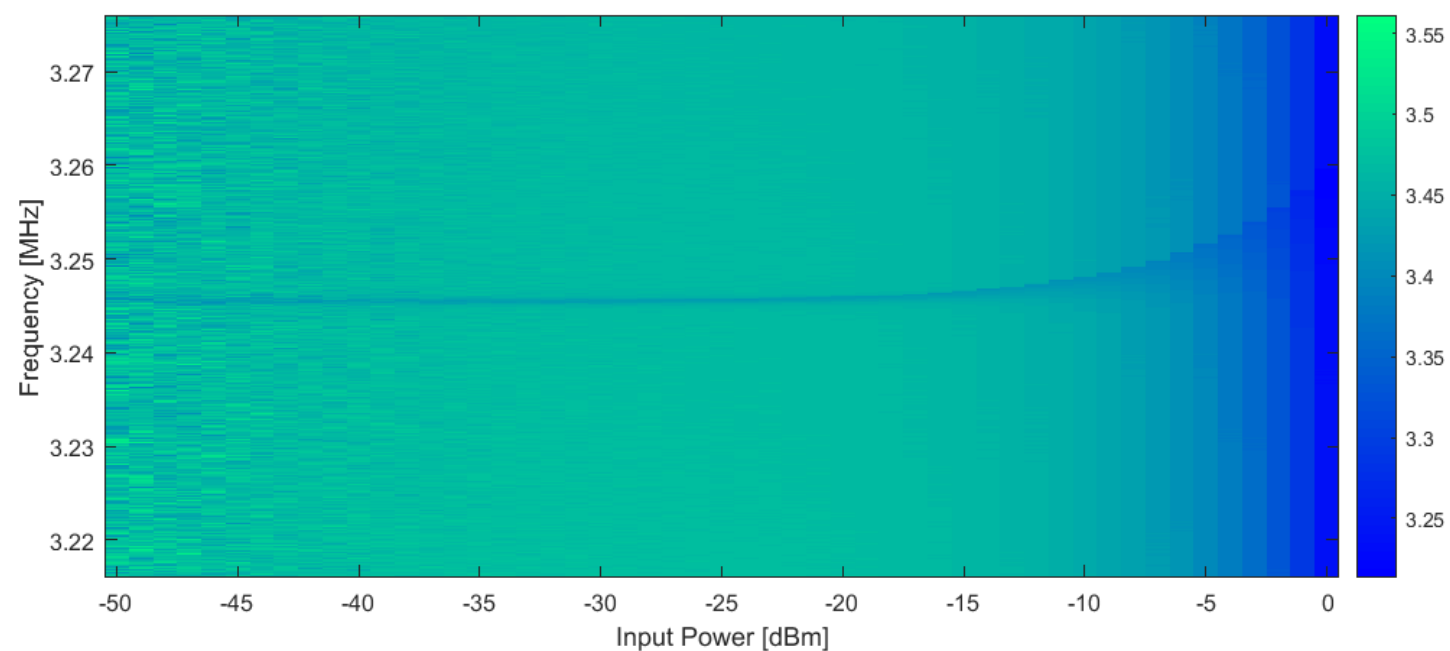

Figure 3. Dependence of the signal transmission coefficient on the frequency and input power of the external signal for nanoresonator with a $2 \mu \mathrm{m}$ length, a $85 \mathrm{~nm}$ width and a $110 \mathrm{~nm}$ height.

\section{CONCLUSIONS}

A well reproducible fabrication technology for nanoscale size resonators based on suspended silicon nanowires made from SOI material is presented. It were fabricated and studied several series of the samples containing nanomechanical systems based on silicon nanowires with the following dimensions: length 2-5 $\mu \mathrm{m}$, height $130 \mathrm{~nm}$, width 50-150 nm. The mechanical characteristics of the nanoresonators were investigated using the magnetomotive method. The proposed method of fabricating of nanomechanical structures uses standard silicon technology. It can be used to produce highly sensitive mass, force and displacement sensors. Also, it can be used as switch for quantum computational networks. The mechanical behavior of the structures was done using a magnetomotive method.

\section{ACKNOWLEDGEMENTS}

The work was supported by the Russian Science Foundation (Grant 16-12-00072). The equipment of the Educational and Methodical Center of Lithography Research Facilities Sharing Centre was used.

\section{REFERENCES}

[1] Ekinci, K.L. and Roukes, M.L., "Nanoelectromechanical systems", Rev. Sci. Instrum. 76, 061101 (2005). https://doi.org/10.1063/1.1927327

[2] Zhang, W.-M., Hu, K.-M., Peng, Z.K., and Meng, G., "Tunable Micro- and Nanomechanical Resonators", Sensors 15(10), 26478-26566 (2015); https://doi.org/10.3390/s151026478

[3] Arash B., Jiang J-W., and Rabczuk T., "A review on nanomechanical resonators and their applications in sensors and molecular transportation", Applied Physics Reviews. 2, 021301 (2015); https://doi.org/10.1063/1.4916728

[4] Greenberg, Ya.S., Pashkin, Yu.A., Il'ichev, E., "Nanomechanical resonators" Phys. Usp. 55, 382-407 (2012). https://doi.org/10.3367/UFNr.0182.201204c.0407

[5] Bartsch, S.T., Arp, M., and Ionescu, A.M., "Junctionless Silicon Nanowire Resonator", IEEE Journal of the Electron Devices Society 2(2), 8-15 (2014); https://doi.org/10.1109/JEDS.2013.2295246

[6] Ilic, B., Yang, Y., and Craighead, H.G., "Virus detection using nanoelectromechanical devices", Appl. Phys. Lett. 85(13), 2604 (2004); https://doi.org/10.1063/1.1794378 
[7] Mamin, H. and Rugar, D., "Sub-attonewton force detection at millikelvin temperatures ", Appl. Phys. Lett. 79, 3358 (2001); https://doi.org/10.1063/1.1418256

[8] Zhao, X., Tsai, J.M., Cai, H., Ji, X.M., Zhou, J., Bao, M.H., Huang, Y.P., Kwong, D.L., and Liu, A.Q., "A nanoopto-mechanical pressure sensor via ring resonator", Opt. Express 20(8), $8535 \quad$ (2012); https://doi.org/10.1364/OE.20.008535

[9] Knobel. R.G. and Cleland, A.N., "Nanometre-scale displacement sensing using a single electron transistor", Nature 424, 291-293 (2003); https://doi.org/10.1038/nature01773

[10] Shevyrin, A.A., Pogosov, A.G., Budantsev, M.V., Bakarov, A.K., Toropov, A.I., Rodyakina, E.E., and Shklyaev, A.A., "Actuation and transduction of resonant vibrations in GaAs/AlGaAs-based nanoelectromechanical systems containing two-dimensional electron gas", Appl. Phys. Lett. 106, 183110 (2015); https://doi.org/10.1063/1.4920932

[11] Naik, A., Buu,O., LaHaye, M.D., Armour, A.D., Clerk, A.A., Blencowe, M.P., and Schwab, K.C., "Cooling a nanomechanical resonator with quantum back-action", Nature 443, 193-196 (2006); http://dx.doi.org/10.1038/nature05027

[12] Teufel, J., Donner, T., Li, D., Harlow, J.W., Allman, M. S., Cicak, K., Sirois, A.J., Wittaker, J.D., Lehnert, K.W., and Simmonds, R.W., "Sideband cooling of micromechanical motion to the quantu", Nature 475, 359-363 (2011); http://dx.doi.org/10.1038/nature10261

[13] Harrabi, K., Pashkin, Yu.A., Astafiev, O.V., Kafanov, S., Li, T.F., and Tsai, J.S., "Fabrication and characterization of Al nanomechanical resonators for coupling to nanoelectronic devices", Appl. Phys. A 108(1), 7-11 (2012); https://doi.org/10.1007/s00339-012-6981-8

[14] Bradley, D.I., George, R., Gu'enault, A.M., Haley, R.P., Kafanov, S., Noble, M.T., Pashkin, Yu.A., Pickett, G.R., Poole, M., Prance, J.R., Sarsby, M., Schanen, R., Tsepelinб V., Wilcox, T., Zmeev, D.E., "Operating Nanobeams in a Quantum Fluid", Scientific Reports, 7, 4876 (2017); https://doi.org/10.1038/s41598-017-04842-y

[15] Loh, O.Y., and Espinosa, H. D., "Nanoelectromechanical contact switches", Nature Nanotechnology, 7, 283-295 (2012); http://dx.doi.org/10.1038/nnano.2012.40

[16] Hwang, K.M., Park, J.Y., Bae, H., Lee, S.W., Kim, C.K., Seo, M., Im, H., Kim, D.H., Kim, S.Y., Lee, G.B., Choi, Y.K., "Nano-electromechanical Switch Based on a Physical Unclonable Function for Highly Robust and Stable Performance in Harsh Environments", ACS Nano., 11(12), $12547-12552$ (2017). https://doi.org/10.1021/acsnano.7b06658

[17] Mori, K., "Silicon-On-Insulator (SOI) Technology", Woodhead Publishing, Singapore, ch. 14, 435-453 (2014), https://doi.org/10.1533/9780857099259.2.435

[18] Dagesyan, S.A., Shorokhov, V.V., Presnov, D.E., Soldatov, E.S., Trifonov, A.S., "Sequential reduction of the silicon single-electron transistor structure to atomic scale", Nanotechnology, 28(22), 225304, (2017). http://dx.doi.org/10.1088/1361-6528/aa6dea

[19] Presnov, D.E., Amitonov, S.V., Krutitskii, P.A., Kolybasova, V.V., Devyatov, I.A., Krupenin, V.A., and Soloviev, I.I., "A highly pH-sensitive nanowire field-effect transistor based", Beilstein J. Nanotechnol., 4, 330335 (2013); https://doi.org/10.3762/bjnano.4.38

[20] D.E. Presnov, S.V. Amitonov, V.A. Krupenin. Silicon nanowire field effect transistor made of silicon-oninsulator. // Russian Microelectronics, 41(5), 310-313, (2012). https://doi.org/10.1134/S1063739712050034

[21] Presnov, D.E., Kafanov, S., Dorofeev, A.A., Bozhev, I.V., Trifonov, A.S., Pashkin, Yu.A., and Krupenin, V.A., "High quality factor mechanical resonance in a silicon nanowire", JETP Letters (2018). https://doi.org/10.1134/S0021364018190037

[22] Trifonov, A.S., Presnov, D.E., Bozhev, I.V., Evplov, D.A., Desmaris, V., and Krupenin, V. A., "Non-contact scanning probe technique for electric field measurements based on nanowire field-effect transistor", Ultramicroscopy, 179, 33-40 (2017). https://doi.org/10.1016/j.ultramic.2017.03.030

[23] Cleland, A.N., and Roukes, M.L., "Fabrication of high frequency nanometer scale mechanical resonators from bulk Si crystals", Appl. Phys. Lett. 69, 2653 (1996); https://doi.org/10.1063/1.117548 